ESAIM: PS 21 (2017) 394-411

DOI: $10.1051 / \mathrm{ps} / 2017017$
ESAIM: Probability and Statistics

www.esaim-ps.org

\title{
BOOTSTRAPPING PERIODICALLY AUTOREGRESSIVE MODELS *
}

\author{
Gabriela Ciołek ${ }^{1}$ and Pawę Potorski ${ }^{2}$
}

\begin{abstract}
The main objective of this paper is to establish the residual and the wild bootstrap procedures for periodically autoregressive models. We use the least squares estimators of model's parameters and generate their bootstrap equivalents. We prove that the bootstrap procedures for causal periodic autoregressive time series with finite fourth moments are weakly consistent. Finally, we confirm our theoretical considerations by simulations.
\end{abstract}

Mathematics Subject Classification. 62M10, 62F12, 62F40.

Received October 28, 2016. Revised August 10, 2017. Accepted August 21, 2017.

\section{INTRODUCTION}

Periodically correlated time series (PC) have been recently extensively investigated due to numerous applications in real data problems. One of the first applications of PC time series can be found in [32] where PC sequences have been used to model monthly stream flow of the rivers. Since then, PC processes have been applied to model many phenomena in numerous fields, for instance in climatology (see $[8,11,19]$ ), finance and econometrics (see $[4,5,17]$ ) or analysis of genome and biological signals (see $[1,12,25]$ ).

The concept of PC times series comes from Gladyšhev (see [13] for details). The fundamental statistical properties of periodic autoregressive sequences (PAR) has been studied in [18] where moment estimation for PAR sequences were investigated. Pagano in [27] showed the asymptotic normality of parameter estimates based on periodic Yule-Walker equations. Vecchia (see [34]) analyzed the correlation structure and the parameter estimation of the periodic autoregression moving average processes (PARMA) with a focus on the moment estimation and maximum likelihood estimation. Basawa and Lund in [3] have investigated large sample properties of parameter estimates for PARMA models and derived a general limit result for coefficients of such models. Shao and $\mathrm{Ni}$ in [30] have studied the least-squares estimation and ANOVA for PAR series. They showed that the limit results for PARMA from [3] also hold exclusively for PAR sequences. Ursu and Duchesne (see [33]) have generalized limit results from $[3,30]$ to vector periodic autoregressive time series model (PVAR).

There is a vast literature on block bootstrap methods for data which are correlated. The first procedures for time series were introduced in $[6,14,21,23]$. Politis in [29] proposed the block bootstrap for series with seasonal component. The Seasonal Block Bootstrap (SBB) presented in this paper requires that the block of size $b$ must

\footnotetext{
Keywords and phrases. Bootstrap, least squares estimation, periodically autoregressive models, time series.

* This work was supported by a public grant as part of the Investissement d'avenir, project reference ANR-11-LABX-0056-LMH.

1 AGH University of Science and Technology, al. Mickiewicza 30, 30-059 Krakow, Poland;

LTCI, Télécom ParisTech, Université Paris-Saclay, 46 Rue Barrault, 75013 Paris, France. gabrielaciolek@gmail.com

2 AGH University of Science and Technology, al. Mickiewicza 30, 30-059 Krakow, Poland. potorski@agh.edu.pl
} 
be at least of order of the period $d$. Chan et al. in [7] formulated a block bootstrap procedure for periodic time series (also nonstationary). This method relies on dividing the observations within each period into blocks of length $b$ and resampling these blocks so that new periods of pseudo-observations are generated. It is noteworthy, that the block bootstrap procedure proposed in [7] is valid for periodic time series with long periodicities, so this method is not consistent with fixed periodicity (since this procedure implores that the period length $d$ has to tend to infinity as the sample size $n$ increases). Dudek et al. in [9] propose the Generalized Seasonal Block Bootstrap (GSBB) that works also with fixed length periodicities that deals with the problems with inconsistency of the method introduced in [7]. However, all of these block bootstrap methods struggle with the problem of choosing the length of the blocks of data in order to reflect the dependence structure of the original sample. This motivated us to formulate bootstrap methods that do not require a choice of the length of bootstrap blocks. Presented methods are data driven, thus recommended to practitioners.

The paper is organized as follows. In Section 2, we introduce the notation and preliminary assumptions for PAR processes. Next, we derive the least squares estimates of models' parameters. We also discuss briefly the asymptotic properties of parameter estimates and recall the crucial limit result of Shao and Ni (presented in [30]) for these estimates. In Section 3, we show that the residual bootstrap procedure for simple autoregressive processes (AR) from [22] can be generalized to PAR time series. This generalization is feasible mainly due to the fact that PAR models can be considered as different AR models for different seasons $v$. This observation allows us to establish a valid bootstrap procedure for causal PAR processes with finite fourth moments. We construct the least squares estimates of PAR model parameters and utilize limit results from [3] and [30] for those estimates in order to show the weak consistency of proposed residual bootstrap method. In Section 4, we introduce the wild bootstrap procedure for PAR processes and prove its weak consistency. Finally, in Section 5, we present a simulation study which illustrates the theory from the previous two sections.

\section{Preliminaries}

In this section we introduce some notation and formulate preliminary assumptions for periodically autoregressive sequences. We will work with the least squares estimators of PAR parameters which are consistent and asymptotically normal due to the limit results given in [3] by Basawa and Lund and in [30] by Shao and Ni.

\subsection{Assumptions and notation}

The periodic autoregressive model is given by

$$
X_{n T+v}=\sum_{k=1}^{p} \phi_{k}(v) X_{n T+v-k}+\epsilon_{n T+v},
$$

where

$$
\Phi^{\prime}=\left[\phi_{1}(1), \phi_{2}(1), \ldots, \phi_{p}(1), \phi_{1}(2), \ldots, \phi_{p}(2), \ldots, \phi_{1}(T), \ldots, \phi_{p}(T)\right]
$$

denotes the vector of parameters and ' is a transpose. The $\left\{X_{n T+v}\right\}$ denotes the series during the $n$-th cycle $(0 \leq n \leq N-1)$ during $v$-th season $(1 \leq v \leq T)$. The $\left\{\epsilon_{n T+v}\right\}$ is the mean zero white noise with variance of the form $\operatorname{Var}\left(\epsilon_{n T+v}\right)=\sigma_{v}^{2}>0$ for all seasons $v$. It is noteworthy that the other periodic notations for the process (2.1) are used (see for instance [2] and references therein). We have chosen the form (2.1) to emphasize that the process (2.1) is AR difference equation with periodically varying coefficients. Observe that for $T=$ $1,(2.1)$ is AR process.

In the following, the periodic notations will be used interchangeably with the non-periodic ones, namely $\left\{X_{t}\right\},\left\{\epsilon_{t}\right\}$. There is no loss of generality in considering the autoregressive model order equal to $p$ instead of $p(v)$ (for each season) as we can take

$$
p=\max _{1 \leq v \leq T} p(v)
$$


and set

$$
\phi_{k}(v)=0 \quad \text { for } \quad p(v)<k \leq p .
$$

The model given by (2.1) may be written as $T$-variate AR (see also [3] for details). We keep our notation consistent with [3]. The $T$-variate AR is given by the difference equation

$$
\Phi_{0} \vec{X}_{n}-\sum_{k=1}^{p^{*}} \Phi_{k} \vec{X}_{n-k}=\vec{\epsilon}_{n}
$$

where $\vec{X}_{n}=\left[X_{n T+1}, \ldots, X_{n T+T}\right]^{\prime}$ and $\vec{\epsilon}_{n}=\left[\epsilon_{n T+1}, \ldots, \epsilon_{n T+T}\right]^{\prime}$. We denote by $p^{*}=\lceil p / T\rceil$ the order of $T$-variate $\mathrm{AR}$ and write $\lceil y\rceil$ for the smallest integer greater than or equal to $y$. The $T \times T$ autoregressive coefficients of $(2.2)$ are of the form

$$
\left(\Phi_{0}\right)_{i, j}=\left\{\begin{array}{lll}
1 & \text { if } & i=j \\
0 & \text { if } & i<j \\
-\phi_{i-j}(i) & \text { if } & i>j
\end{array}\right.
$$

and

$$
\left(\Phi_{k}\right)_{i, j}=\phi_{k T+i-j}(i), \quad 1 \leq k \leq p^{*}
$$

with the convention $\phi_{k}(v)=0$ for $k>p$.

We say that PAR model is causal if

$$
\operatorname{det}\left(\Phi_{0}-\sum_{k=1}^{p^{*}} \Phi_{k} z^{k}\right) \neq 0
$$

for all complex $z$ such that $|z| \leq 1$. It is known that when the model is causal, then the unique $T$-variate solution $\left\{\vec{X}_{n}\right\}$ to $(2.2)$ is multivariate stationary. Process $\left\{X_{t}\right\}$ is periodically correlated since we have periodic stationarity of the moments, namely

$$
\mathbb{E}\left(X_{n+T}\right)=\mathbb{E}\left(X_{n}\right) \quad \text { and } \quad \operatorname{Cov}\left(X_{n+T}, X_{m+T}\right)=\operatorname{Cov}\left(X_{n}, X_{m}\right) .
$$

Models $X_{t}$ which fulfill above moment conditions are called periodically correlated or cyclostationary (see $[2,13]$ for further details).

\subsection{The least squares estimation for model's parameters}

To generate valid bootstrap approximations we have to obtain the least squares estimators of $\Phi$, namely

$$
\widehat{\Phi}^{\prime}=\left[\hat{\phi}_{1}(1), \hat{\phi}_{2}(1), \ldots, \hat{\phi}_{p}(1), \hat{\phi}_{1}(2), \ldots, \hat{\phi}_{p}(2), \ldots, \hat{\phi}_{1}(T), \ldots, \hat{\phi}_{p}(T)\right] .
$$

We rearrange (2.1) as follows

$$
\epsilon_{n T+v}=X_{n T+v}-\sum_{k=1}^{p} \phi_{k}(v) X_{n T+v-k} .
$$

Next, we minimize the sum of squared errors

$$
S(\Phi)=\sum_{v=1}^{T} \mathrm{e}^{\prime}(v) e(v),
$$


where

$$
e(v)=\left[\epsilon_{v}, \epsilon_{T+v}, \ldots, \epsilon_{(N-1) T+v}\right]
$$

is $1 \times N$ vector of errors. In order to obtain the least squares estimator of $\Phi$ we differentiate $S(\Phi)$ with respect to each parameter $\phi_{k}(v), k=1, \ldots, p(v) ; v=1, \ldots, T$. Thus, we have

$$
\frac{\partial S(\Phi)}{\partial \phi_{k}(v)}=-2 \sum_{n=0}^{N-1} X_{n T+v-k} \epsilon_{n T+v} .
$$

Next, we set the derivatives equal to zero (for $k=1, \ldots, p(v))$ and obtain for a given season $v$

$$
\sum_{n=0}^{N-1} W_{n}(v) \epsilon_{n T+v}=\overrightarrow{0}
$$

where $\overrightarrow{0}$ is $p(v) \times 1$ vector of zeros and

$$
W_{n}(v)=\left[X_{n T+v-1}, \ldots, X_{n T+v-p(v)}\right]^{\prime}
$$

are $p(v) \times 1$ random vectors, $n=0,1, \ldots, N-1$. Vectors defined in (2.5) form a $N \times p(v)$ random matrix

$$
W(v)=\left[W_{0}(v), \ldots, W_{N-1}(v)\right]^{\prime} .
$$

We consider

$$
\epsilon_{n T+v}=X_{n T+v}-W_{n}^{\prime}(v) \Phi(v),
$$

where $\Phi(v)=\left[\phi_{1}(v), \ldots, \phi_{p(v)}(v)\right]^{\prime}$. The normal equations for (2.6) at the season $v$ are of the form

$$
\sum_{n=0}^{N-1} W_{n}(v) X_{n T+v}=\left(\sum_{n=0}^{N-1} W_{n}(v) W_{n}^{\prime}(v)\right) \Phi(v) .
$$

The least squares estimators of $\Phi(v)$ satisfy the following relation

$$
\widehat{\Phi}(v)=\left(\left(W^{\prime}(v) W(v)\right)^{-1} W^{\prime}(v)\right) z(v),
$$

where

$$
z(v)=\left[X_{v}, X_{T+v}, \ldots, X_{(N-1) T+v}\right]^{\prime}
$$

is $N \times 1$ random vector and

$$
\widehat{\Phi}(v)=\left[\phi_{1}(v), \ldots, \phi_{p(v)}(v)\right]^{\prime}
$$

is $p(v) \times 1$ vector of the least squares estimates of parameters of model $(2.6)$ at the season $v, v=1, \ldots, T$. The invertibility of $W^{\prime}(v) W(v)$ is ensured by Proposition 4.1 from [2]. For the sake of completeness, we recall this proposition below.

Remark 2.1. If $\sigma_{v}>0$ for each $1 \leq v \leq T$, then for a causal PAR model, $W^{\prime}(v) W(v)$ is invertible for each $n \geq 1$.

Thus, the residuals are of the form

$$
\hat{\epsilon}_{n T+v}=X_{n T+v}-\left(W_{n}^{\prime}(v) \widehat{\Phi}(v)\right) .
$$

In the next subsection we discuss the asymptotic normality of $\widehat{\Phi}(v), 1 \leq v \leq T$. This result is essential for our bootstrap theory to work. 


\subsection{Asymptotic properties of parameters' estimates}

In order to establish a bootstrap procedure for (2.6), we make use of the theorems from [3,30]. For completeness of exposition, we now recall the theorem of Shao and Ni from [30] which is the general limit result for $\operatorname{PAR}(p)$ process. In the following we denote by $\stackrel{D}{\longrightarrow}$ the convergence in distribution.

Theorem 2.2. Assume that a periodic autoregressive series $\left\{X_{n T+v}\right\}$ defined in (2.1) is causal with finite fourth moment. Then,

$$
\sqrt{N}(\widehat{\Phi}-\Phi) \stackrel{D}{\rightarrow} \mathcal{N}\left(0, F^{-1}\right) \text { as } n \rightarrow \infty,
$$

where $F=F_{\phi}\left(v_{1}, v_{2}\right)$ and is defined in the following way: for any $k \geq 1, l \leq p$ and $v_{1} \geq 1, v_{2} \leq T$

$$
F_{\phi}\left(v_{1}, v_{2}\right)=\mathbb{E}\left(\left(\frac{\partial \tilde{\epsilon_{n}}}{\partial \phi_{k}\left(v_{1}\right)}\right)\left(\frac{\partial \tilde{\epsilon_{n}}}{\partial \phi_{l}\left(v_{2}\right)}\right)^{\prime}\right),
$$

where

$$
\tilde{\epsilon}^{\prime}=\left[\tilde{\epsilon}_{0}^{\prime}, \tilde{\epsilon}_{1}^{\prime}, \ldots, \tilde{\epsilon}_{N-1}^{\prime}\right], \quad \tilde{\epsilon}_{n}^{\prime}=\left[\frac{\epsilon_{n T+1}}{\sigma_{1}}, \frac{\epsilon_{n T+2}}{\sigma_{2}}, \ldots, \frac{\epsilon_{n T+T}}{\sigma_{T}}\right]
$$

and $\sigma_{v}$ is the standard deviation of $\epsilon_{n T+v}$ at the season $v(1 \leq v \leq T)$.

Remark 2.3. In the above theorem we assumed that $\sigma_{v}$ is known for each season $v(1 \leq v \leq T)$. However, if $\sigma_{v}$ is unknown, it can be replaced by $\sqrt{N}$-consistent estimator. The limiting distribution of $\widehat{\Phi}$ remains unchanged (see [3] for details).

\section{Bootstrap VAlidity For $\operatorname{PAR}(p)$ Process}

In this section we formulate the residual and the wild bootstrap procedures for $\operatorname{PAR}(p)$ processes in order to define confidence intervals of parameters and validate the model given by (2.1). We have decided to work with those methods in order to avoid block resampling when dealing with dependent random variables $X_{i}$ 's (see [22] for details). It is noteworthy that the residual bootstrap method is designed for heteroscedastic models, however we adapt it in order to make it applicable for PAR sequences. The second method we present is the wild bootstrap which is tailor-made for heteroscedastic models. In both methods, the first step is to obtain the ordinary least squares estimator $\hat{\Phi}$ of $\Phi$ and secondly to compute residuals

$$
\hat{\epsilon}_{n T+v}=X_{n T+v}-\sum_{i=1}^{p} \hat{\phi}_{k}(v) X_{n T+v-k} .
$$

The difference between those two methods relies on different way of computing bootstrap version of residuals. In residual bootstrap one draws randomly with replacement centred and scaled residuals in order to get bootstrap random variables $\bar{\eta}_{n T+v}^{*}$. In order to have the same periodic structure of the bootstrap version of $X_{n T+v}$, we multiply $\bar{\eta}_{n T+v}^{*}$ by standard deviation of corresponding season and $X_{n T+v}$. Finally, we generate the bootstrap version $X_{n T+v}^{*}$ of PAR process and compute bootstrap estimates $\hat{\Phi}^{*}$. In the wild bootstrap method we obtain bootstrap version of residuals (3.1) by multiplying $\hat{\epsilon}_{n T+v}$ by random variables drawn from normal distribution with zero mean and variance 1 . From now, we generate bootstrap version of process $X_{n T+v}$ with bootstrap residuals $\epsilon_{n T+V}^{\dagger}$. Thus, the wild bootstrap method results in obtaining bootstrap estimates $\hat{\Phi}^{\dagger}$.

Usually, one uses the wild bootstrap when there is heteroscedasticity in the model. Residual bootstrap method is designed for models that are homoscedastic. PAR models are heteroscedastic, thus a natural way is to use the wild bootstrap method. However, in our paper we adapt residual bootstrap procedure (by diving residuals by $\sigma_{v}$ in Step 4 and multiplying residuals by $\sigma_{v}$ in Step 5) such that it can be used for PAR sequences. 
It is noteworthy that from a second order theory point of view (see [15]) the residual bootstrap outperforms the wild bootstrap (generated by Gaussian noise). Indeed, the second one can not correct adequately the skewness of the distribution. However, in practice, this difference between two methods is difficult to distinguish (except for very small samples, see our simulation studies in the last chapter).

The bootstrap procedure for $\operatorname{PAR}(p)$ processes is formulated as follows.

\section{Algorithm 3.1.}

(1) Compute the ordinary least squares estimator $\widehat{\Phi}$ of $\Phi$.

(2) Compute the residuals of the estimated model

$$
\hat{\epsilon}_{n T+v}=X_{n T+v}-\sum_{k=1}^{p} \hat{\phi}_{k}(v) X_{n T+v-k},
$$

where $1 \leq v \leq T, 0 \leq n \leq N-1$.

(3) Compute the centred residuals

$$
\bar{\eta}_{n T+v}=\frac{\hat{\epsilon}_{n T+v}}{\sigma_{v}}-\frac{1}{N T} \sum_{n=0}^{N-1} \sum_{v=1}^{T} \frac{\hat{\epsilon}_{n T+v}}{\sigma_{v}},
$$

where NT is the number of all observations in the model.

Remark 3.2. In the case when $\sigma_{v}^{2}$ are known, we divide $\hat{\epsilon}_{n T+v}$ by the true standard deviation $\sigma_{v}$. When $\sigma_{v}^{2}$ are unknown, we replace it by a $\sqrt{N}$-consistent estimator of $\sigma_{v}^{2} ;$ e.g., one can use the sample variance of the residuals obtained in the second step of Algorithm 3.1, that is from sample $\left\{\hat{\epsilon}_{n T+v}\right\}_{n=0}^{N-1}$.

(4) Generate bootstrap variables $\eta_{n T+v}^{*}$ by drawing randomly with replacement from $\left\{\bar{\eta}_{1}, \ldots, \bar{\eta}_{N T}\right\}$.

Remark 3.3. Note that $\eta_{n T+v}^{*}, 0 \leq n \leq N-1,1 \leq v \leq T$ are conditionally independent on the data. Their common distribution is defined as

$$
\mathbb{P}^{*}\left(\eta_{1}^{*}=\bar{\eta}_{n T+v}\right)=\frac{1}{N T} .
$$

(5) Generate the bootstrap version of the model (2.1)

$$
X_{n T+v}^{*}=\sum_{k=1}^{p} \hat{\phi}_{k}(v) X_{n T+v-k}+\sigma_{v} \eta_{n T+v}^{*},
$$

$1 \leq v \leq T$.

(6) Calculate the bootstrap estimators of parameters for each season $v, 1 \leq v \leq T$

$$
\widehat{\Phi}^{*}(v)=\left(W^{\prime}(v) W(v)\right)^{-1} W^{\prime}(v) z^{*}(v),
$$

where

$$
z^{*}(v)=\left[X_{v}^{*}, \ldots, X_{(N-1) T+v}^{*}\right]^{\prime}, 1 \leq v \leq T .
$$

Remark 3.4. Note that $\widehat{\Phi}^{*}$ can be written as

$$
\begin{aligned}
\widehat{\Phi}^{*}(v) & =\left(W^{\prime}(v) W(v)\right)^{-1} W^{\prime}(v)\left(W(v) \widehat{\Phi}(v)+g^{*}(v)\right) \\
& =\widehat{\Phi}(v)+\left(W^{\prime}(v) W(v)\right)^{-1} W^{\prime}(v) g^{*}(v),
\end{aligned}
$$

where

$$
g^{*}(v)=\left[\epsilon_{v}^{*}, \ldots, \epsilon_{(N-1) T+v}^{*}\right]^{\prime}=\left[\sigma_{v} \eta_{v}^{*}, \ldots, \sigma_{v} \eta_{(N-1) T+v}^{*}\right]^{\prime} .
$$




\subsection{Consistency of the bootstrap procedure}

In this subsection we prove that the bootstrap procedure for a periodic autoregressive series $X_{n T+v}$ formulated in the Section 3 is weakly consistent.

Definition 3.5. We say that the bootstrap procedure is weakly consistent if the distribution of the bootstrap approximation $\mathcal{L}\left(\sqrt{N}\left(\widehat{\Phi}^{*}-\widehat{\Phi}\right)\right)$ converges weakly in probability to the same distribution as the distribution of the estimation error of the original estimator $\mathcal{L}(\sqrt{N}(\widehat{\Phi}-\Phi))$.

The proof of the validity of bootstrap is kept in spirit of the proof of Theorem 3.1 from [31]. We denote by $\stackrel{P^{*}}{\longrightarrow}$ weak convergence conditionally on the data in probability.

Theorem 3.6. Suppose that a periodic autoregressive series $X_{n T+v}$ defined in (2.1) is causal and has finite fourth moment. Then, the residual bootstrap procedure given by the Algorithm 3.1 is weakly consistent, i.e.

$$
\sqrt{N}\left(\widehat{\Phi}^{*}-\widehat{\Phi}\right) \stackrel{P^{*}}{\longrightarrow} \mathcal{N}\left(0, F^{-1}\right) .
$$

Proof. The theorem from [30] guarantees that

$$
\sqrt{N}(\widehat{\Phi}-\Phi) \stackrel{D}{\longrightarrow} \mathcal{N}\left(0, F^{-1}\right)
$$

where

$$
F=F_{\phi}\left(v_{1}, v_{2}\right)=\mathbb{E}\left(\frac{\partial \tilde{\epsilon}_{n}}{\partial \phi_{k}\left(v_{1}\right)} \cdot \frac{\partial \tilde{\epsilon}_{n}}{\partial \phi_{l}\left(v_{2}\right)}\right), 1 \leq v_{1}, v_{2} \leq T
$$

and

$$
\tilde{\epsilon}_{n}^{\prime}=\left[\frac{\epsilon_{n T+1}}{\sigma_{1}}, \frac{\epsilon_{n T+2}}{\sigma_{2}}, \ldots, \frac{\epsilon_{n T+T}}{\sigma_{T}}\right]
$$

and $\sigma_{v}$ is the standard deviation of $\epsilon_{n T+v}$ for $1 \leq v \leq T$.

Moreover, in [30] Shao and Ni have shown that the estimates of $\Phi\left(v_{1}\right)$ and $\Phi\left(v_{2}\right)$ are asymptotically independent if $v_{1} \neq v_{2}$. It follows from Remark 3.4 that

$$
\widehat{\Phi}^{*}(v)-\widehat{\Phi}(v)=\left(W^{\prime}(v) W(v)\right)^{-1} W^{\prime}(v) g^{*}(v) .
$$

Note, that the analysis of the $\operatorname{PAR}(p)$ model corresponds to the analysis of $\operatorname{AR}(p(v))$ model for each fixed $v(1 \leq v \leq T)$, respectively. This observation allows us to apply directly the asymptotic results from [16]. Note however, that our method is more general and the proof based on [30] also allows us to use dependent heteroscedastic residuals.

By the Hamilton's remarks (see [16], Chap. 8, p. 215), we have for a given $v, 1 \leq v \leq T$,

$$
\frac{1}{N} W^{\prime}(v) W(v) \stackrel{P}{\rightarrow} F
$$

where $\stackrel{P}{\longrightarrow}$ denotes convergence in probability. Thus, by the virtue of (3.4) combined with the Slutsky theorem, it remains to prove that

$$
\frac{1}{\sqrt{N}} W^{\prime}(v) g^{*}(v) \stackrel{P^{*}}{\longrightarrow} \mathcal{N}\left(0, \sigma_{v}^{2} F\right) .
$$

Note that for a given season $v$ we have

$$
\frac{1}{\sqrt{N}} W^{\prime}(v) g^{*}(v)=\frac{1}{\sqrt{N}} \sum_{n=0}^{N-1} \epsilon_{n T+v}^{*}(v) W_{n}(v) .
$$


We introduce one more piece of notation. We write

$$
y_{n}^{*}=\frac{\epsilon_{n T+v}^{*} W_{n}(v)}{\sqrt{N}} .
$$

In order to show the consistency of bootstrap procedure formulated in 3.1, we check whether the conditions of Lyapunov's central limit theorem hold. Firstly, observe that

$$
\mathbb{E}^{*}\left(y_{n}^{*}\right)=\frac{1}{\sqrt{N}} W_{n}(v) \mathbb{E}\left(\epsilon_{n T+v}^{*}\right)=0,
$$

because $\epsilon_{n T+v}^{*}=\sigma(v) \eta_{n T+v}^{*}$ and $\mathbb{E}\left(\eta_{n T+v}^{*}\right)=0$ since $\left\{\eta_{n T+v}^{*}\right\}_{0 \leq n \leq N-1}$ are drawn from the empirical distribution function given by (3.2). Next, we have

$$
\sum_{n=0}^{N-1} \mathbb{E}^{*}\left(y_{n}^{*} y_{n}^{* \prime}\right)=\frac{1}{N} \sum_{n=0}^{N-1} W_{n}(v) W_{n}^{\prime}(v) \mathbb{E}^{*}\left(\epsilon_{n T+v}^{* 2}\right) .
$$

For a given season $v$ we obtain

$$
\begin{aligned}
\mathbb{E}^{*}\left(\epsilon_{n T+v}^{* 2}\right) & =\frac{1}{N} \sum_{n=0}^{N-1} \sigma_{v}^{2} \eta_{n}^{* 2}(v) \\
& =\frac{1}{N} \sum_{n=0}^{N-1}\left(\widehat{\epsilon}_{n}(v)-\bar{\epsilon}\right)^{2} \\
& =\frac{1}{N} \sum_{n=0}^{N-1} \widehat{\epsilon}_{n}^{2}(v)-\bar{\epsilon}^{2} \\
& =\mathbb{E}\left(\epsilon_{n}^{2}(v)\right)-\mathbb{E}^{2}\left(\epsilon_{n}(v)\right)+o_{\mathbb{P}}(1),
\end{aligned}
$$

where

and

$$
\eta_{n}^{*}(v)=\left[\eta_{n T+v-1}^{*}, \ldots, \eta_{n T+v-p(v)}^{*}\right]^{\prime}
$$

$$
\widehat{\epsilon}_{n}(v)=\left[\hat{\epsilon}_{n T+v-1}, \ldots, \hat{\epsilon}_{n T+v-p(v)}\right]^{\prime} .
$$

Thus, by (3.4) and (3.5) we can deduce that

$$
\sum_{n=0}^{N-1} \mathbb{E}^{*}\left(y_{n}^{*} y_{n}^{* \prime}\right)=\frac{1}{N} \sum_{n=0}^{N-1} W_{n}(v) W_{n}^{\prime}(v) \mathbb{E}^{*}\left(\epsilon_{n T+v}^{* 2}\right) \stackrel{P}{\rightarrow} \sigma_{v}^{2} F .
$$

Let $c \in \mathbb{R}^{p}$ and

$$
s_{N}^{2}=\sum_{n=0}^{N-1} \mathbb{E}^{*}\left(c^{\prime} y_{n}^{*}\right)^{2} \stackrel{P}{\longrightarrow} c^{\prime} \sigma_{v}^{2} F c
$$

by (3.6) and the Cramér-Wold device. We check the Lyapunov's condition for $\gamma=1$.

$$
\begin{aligned}
\frac{1}{s_{N}^{3}} \sum_{n=0}^{N-1} \mathbb{E}^{*}\left|c^{\prime} y_{n}^{*}\right|^{3} & =\frac{1}{s_{N}^{3}} \sum_{n=0}^{N-1} \frac{1}{N^{3 / 2}} \mathbb{E}^{*}\left|\epsilon_{n T+v}^{*}\right|^{3}\left|c^{\prime} W_{n}(v)\right|^{3} \\
& =\frac{1}{s_{N}^{3}} \sum_{n=0}^{N-1} \frac{1}{\sqrt{N}} \mathbb{E}^{*}\left|\epsilon_{n T+v}^{*}\right|^{3} \frac{1}{N}\left|c^{\prime} W_{n}(v)\right|^{3} .
\end{aligned}
$$


Notice, that by the similar arguments as in (3.5) we have for a given $v$

$$
\mathbb{E}^{*}\left|\epsilon_{n T+v}^{*}\right|^{3}=\frac{1}{N} \sum_{n=0}^{N-1}\left|\widehat{\epsilon}_{n}(v)-\bar{\epsilon}\right|^{3}=o_{\mathbb{P}}(1) .
$$

Moreover,

$$
\frac{1}{N}\left|c^{\prime} W_{n}(v)\right|^{3}=O_{\mathbb{P}}(1)
$$

since we assumed that $X_{n T+v}$ has finite fourth moment.

In a view of above discussion, we conclude that

$$
\frac{1}{s_{N}^{3}} \sum_{n=0}^{N-1} \mathbb{E}^{*}\left|c^{\prime} y_{n}^{*}\right|^{3}=o_{\mathbb{P}}(1) .
$$

We have checked that the Lyapunov's conditions for the central limit theorem hold. Next, consider the sequence $c_{0}^{\prime} y_{0}^{*}, \ldots, c_{N-1}^{\prime} y_{N-1}^{*}$. We apply to that sequence the Cramér-Wold theorem and the central limit theorem for triangular arrays. Thus, we obtain the following convergence

$$
\frac{1}{\sqrt{N}} \sum_{n=0}^{N-1} y_{n}^{*} \stackrel{P^{*}}{\longrightarrow} \mathcal{N}\left(0, \sigma_{v}^{2} F\right) .
$$

Combining (3.4) and (3.10) with the Slutsky theorem we obtain immediately that

$$
\sqrt{N}\left(\widehat{\Phi}^{*}-\widehat{\Phi}\right) \stackrel{P^{*}}{\longrightarrow} \mathcal{N}\left(0, F^{-1}\right)
$$

taking scaled versions of $\left\{\epsilon_{n}^{\prime}\right\}_{0 \leq n \leq N-1}$ and $\left\{\epsilon_{n}^{* \prime}\right\}_{0 \leq n \leq N-1}$, namely

$$
\tilde{\epsilon}_{n}^{\prime}=\left[\frac{\epsilon_{n T+1}}{\sigma_{1}}, \ldots, \frac{\epsilon_{n T+T}}{\sigma_{T}}\right]
$$

and

$$
\tilde{\epsilon}_{n}^{* \prime}=\left[\frac{\epsilon_{n T+1}^{*}}{\sigma_{1}}, \ldots, \frac{\epsilon_{n T+T}^{*}}{\sigma_{T}}\right]
$$

It is easy to see that Theorem 3.6 generalizes the results for AR processes (compare with Chap. 8 in [22]). In the next section we present simulation study which demonstrates the large sample properties of the residual bootstrap for PAR sequences.

\section{THE WILD BOOTSTRAP}

In this section we present the wild bootstrap method for periodically autoregressive models as an alternative to the residual bootstrap procedure from the previous section. We investigate the behaviour of those two methods through simulations study in Section 5. As mentioned before, in the wild boostrap procedure one obtains the bootstrap version of residuals $\epsilon_{n T+v}^{\dagger}$ by multiplying the residuals $\hat{\epsilon}_{n T+v}$ by realizations from normal distribution $\mathcal{N}(0,1)$. This procedure is described for example in [20] or [23]. The wild bootstrap procedure for $\operatorname{PAR}(p)$ processes is formulated as follows.

\section{Algorithm 4.1.}

(1) Compute the ordinary least squares estimator $\widehat{\Phi}$ of $\Phi$. 
(2) Compute the residuals of the estimated model

$$
\hat{\epsilon}_{n T+v}=X_{n T+v}-\sum_{k=1}^{p} \hat{\phi}_{k}(v) X_{n T+v-k},
$$

where $1 \leq v \leq T, 0 \leq n \leq N-1$.

(3) Generate the bootstrap process $X_{n T+v}^{\dagger}$ for each season $v, 1 \leq v \leq T$

$$
X_{n T+v}^{\dagger}=\sum_{k=1}^{p} \hat{\phi}_{k}(v) X_{n T+v-k}+\epsilon_{n T+v}^{\dagger}
$$

and

$$
\epsilon_{n T+v}^{\dagger}=\hat{\epsilon}_{n T+v} \eta_{n T+v}^{\dagger}
$$

where $\eta_{n T+v}^{\dagger} \sim \mathcal{N}(0,1)$ and $\left(\eta_{n T+v}^{\dagger}\right)_{n T+v \in \mathbb{R}}$ is independent of $\hat{\epsilon}_{n T+v}$.

(4) Calculate the bootstrap estimator of parameters, namely

$$
\widehat{\Phi}^{\dagger}(v)=\left(W^{\prime}(v) W(v)\right)^{-1} W^{\prime}(v) z^{\dagger}(v)
$$

for each season $v, 1 \leq v \leq T$, where

$$
z^{\dagger}(v)=\left[X_{v}^{\dagger}, \ldots, X_{(N-1) T+v}^{\dagger}\right]^{\prime}
$$

Remark 4.2. Note that $\widehat{\Phi}^{\dagger}$ can be written as

$$
\begin{aligned}
\widehat{\Phi}^{\dagger}(v) & =\left(W^{\prime}(v) W(v)\right)^{-1} W^{\prime}(v)\left(W(v) \widehat{\Phi}(v)+g^{\dagger}(v)\right) \\
& =\widehat{\Phi}(v)+\left(W^{\prime}(v) W(v)\right)^{-1} W^{\prime}(v) g^{\dagger}(v),
\end{aligned}
$$

where

$$
g^{\dagger}(v)=\left[\epsilon_{v}^{\dagger}, \ldots, \epsilon_{(N-1) T+v}^{\dagger}\right]^{\prime}=\left[\hat{\epsilon}_{v} \eta_{v}^{\dagger}, \ldots, \hat{\epsilon}_{(N-1) T+v} \eta_{(N-1) T+v}^{\dagger}\right]^{\prime} .
$$

\subsection{Consistency of the wild bootstrap procedure}

In this subsection we show weak consistency of the wild bootstrap procedure for PAR sequences formulated in Algorithm 4.1.

Theorem 4.3. Assume that a periodic autoregressive series $X_{n T+v}$ defined in (2.1) is causal and has finite fourth moment. Then, the wild bootstrap procedure defined in Algorithm 4.1 is weakly consistent, i.e.

$$
\sqrt{N}\left(\widehat{\Phi}^{\dagger}-\widehat{\Phi}\right) \stackrel{P^{*}}{\longrightarrow} \mathcal{N}\left(0, F^{-1}\right)
$$

where

$$
F=F_{\phi}\left(v_{1}, v_{2}\right)=\mathbb{E}\left(\frac{\partial \tilde{\epsilon}_{n}}{\partial \phi_{k}\left(v_{1}\right)} \cdot \frac{\partial \tilde{\epsilon}_{n}}{\partial \phi_{l}\left(v_{2}\right)}\right), 1 \leq v_{1}, v_{2} \leq T
$$

and

$$
\tilde{\epsilon}_{n}^{\prime}=\left[\frac{\epsilon_{n T+1}}{\sigma_{1}}, \frac{\epsilon_{n T+2}}{\sigma_{2}}, \ldots, \frac{\epsilon_{n T+T}}{\sigma_{T}}\right]
$$

and $\sigma_{v}$ is the standard deviation of $\epsilon_{n T+v}$ for $1 \leq v \leq T$.

Proof. The proof of Theorem 4.3 is analogous to the proof of Theorem 3.6 and it comes down to checking conditions of Lyapunov's central limit theorem. 


\section{Simulations}

The purpose of these simulations is to check the performance of the proposed procedures, namely the residual and the wild bootstrap for PAR processes. Firstly, we compare densities of $\sqrt{N}\left(\widehat{\Phi}^{*}-\widehat{\Phi}\right)$ and $\sqrt{N}(\widehat{\Phi}-\Phi)$. Secondly, we compare densities $\sqrt{N}\left(\widehat{\Phi}^{\dagger}-\widehat{\Phi}\right)$ and $\sqrt{N}(\widehat{\Phi}-\Phi)$. Next, we compute actual coverage probabilities (ACP) for simulated PAR sequences. We perform simulations for three different $\operatorname{PAR}(p)$ processes in order to show that both methods work well for simple and more complicated models with different number of periods and seasons. In the residual bootstrap procedure in the third step of Algorithm 3.1 we used the sample variance of the residuals obtained in the second step of Algorithm 3.1 since for simulations we assume that $\sigma_{v}^{2}$ are unknown (see Rem. 3.2). We consider the following PAR models:

$\operatorname{M1} \operatorname{PAR}(1), T=3, \sigma_{1}=1, \sigma_{2}=1, \sigma_{3}=1, \phi_{1}(1)=0.2, \phi_{1}(2)=0.3, \phi_{1}(3)=0.2$,

M2 $\operatorname{PAR}(3), T=3, \sigma_{1}=1, \sigma_{2}=1, \sigma_{3}=1, \phi_{1}(1)=0.2, \phi_{2}(1)=0.1, \phi_{3}(1)=0.05, \phi_{1}(2)=0.3, \phi_{2}(2)=0.2$, $\phi_{3}(2)=0.1, \phi_{1}(3)=0.2, \phi_{2}(3)=0.1, \phi_{3}(3)=0.05$,

M3 $\operatorname{PAR}(2), T=2, \sigma_{1}=1, \sigma_{2}=2, \phi_{1}(1)=0.2, \phi_{2}(1)=0.1, \phi_{1}(2)=0.3, \phi_{2}(2)=0.2$,

In our simulations we take 599 bootstrap repetitions. We observe that both bootstrap methods work well for $n=100$ and larger samples, below we illustrate the case when $n=300$. Our simulations revealed that the residual and the wild bootstrap methods struggle when sample size $n<100$. We show that the wild bootstrap method behaves worse than the residual bootstrap when the samples are small, below we give an example for $n=30$.

Figures 1-6 show that for all considered models tails of distributions are well estimated (when applying the residual and the wild bootstrap procedures). Figures show that especially for the residual bootstrap the centres

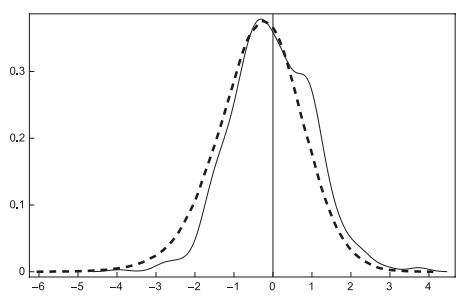

(a) $k=1, v=1$

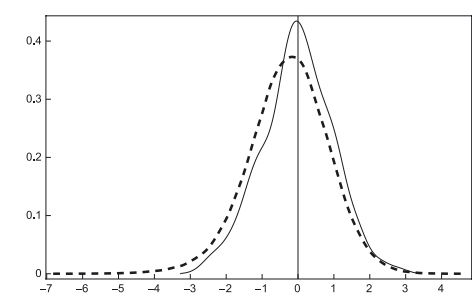

(b) $k=1, v=2$

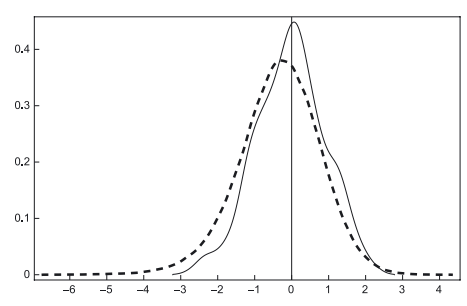

(c) $k=1, v=3$

FiguRE 1. Comparison of performance: the residual bootstrap (thick dashed lines: $\sqrt{N}\left(\widehat{\phi}_{k}^{*}(v)-\right.$ $\left.\widehat{\phi}_{k}(v)\right)$ ) and the least squares estimators (thin lines: $\sqrt{N}\left(\widehat{\phi}_{k}(v)-\phi_{k}(v)\right)$ ) for M1 and sample size $n=300$.

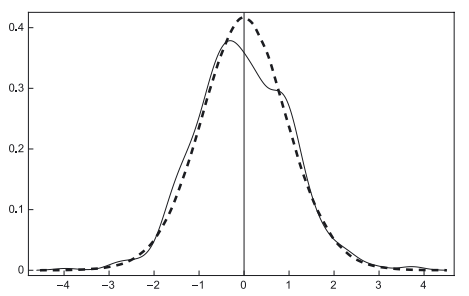

(a) $k=1, v=1$

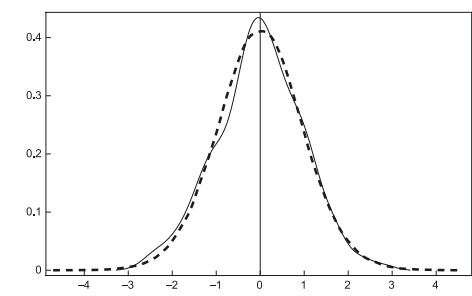

(b) $k=1, v=2$

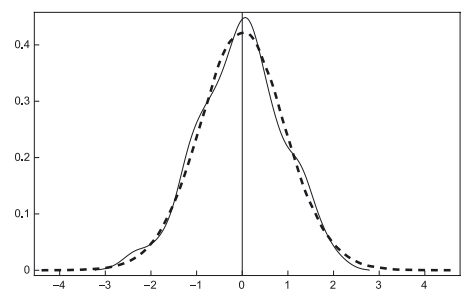

(c) $k=1, v=3$

FIGURE 2. Comparison of performance: the wild bootstrap (thick dashed lines: $\sqrt{N}\left(\widehat{\phi}_{k}^{\dagger}(v)-\right.$ $\left.\widehat{\phi}_{k}(v)\right)$ ) and the least squares estimators (thin lines: $\sqrt{N}\left(\widehat{\phi}_{k}(v)-\phi_{k}(v)\right)$ ) for M1 and sample size $n=300$. 


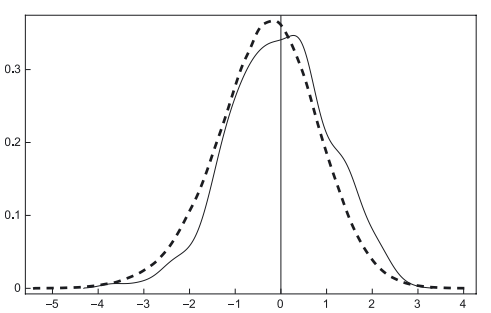

(a) $k=1, v=1$

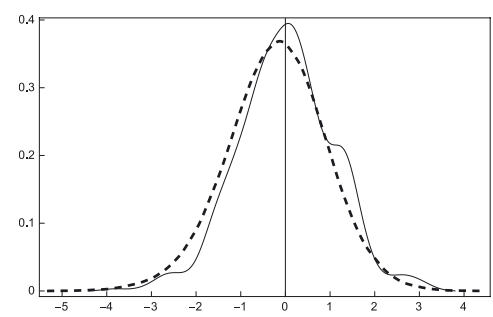

(d) $k=1, v=2$

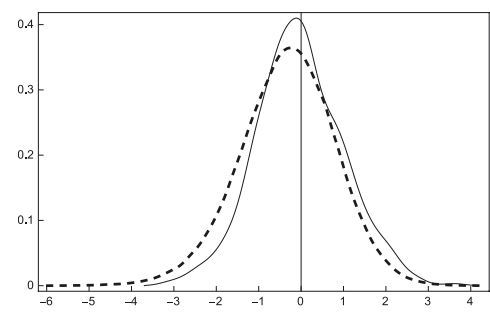

(g) $k=1, v=3$

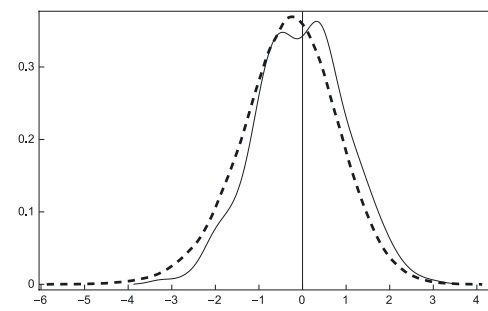

(b) $k=2, v=1$

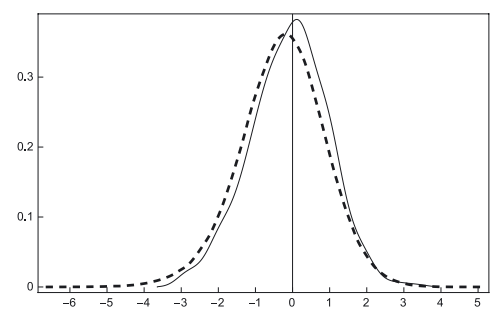

(e) $k=2, v=2$

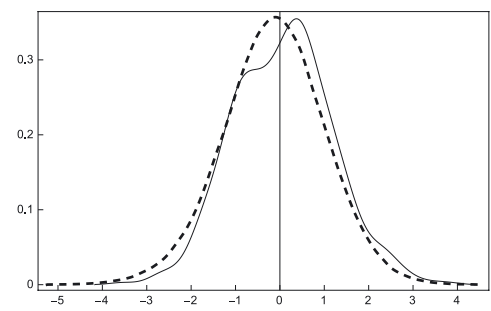

(h) $k=2, v=3$

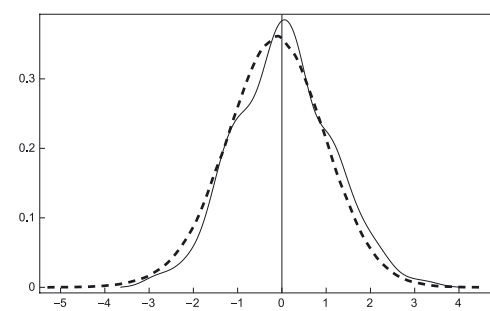

(c) $k=3, v=1$

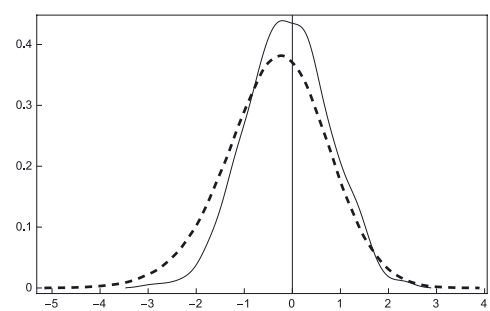

(f) $k=3, v=2$

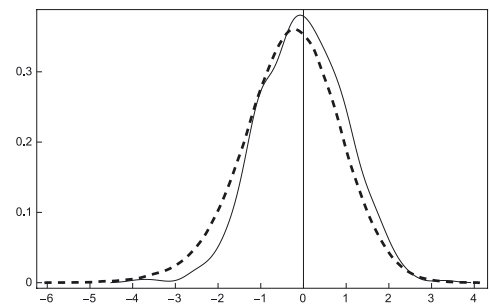

(i) $k=3, v=3$

FIGURE 3. Comparison of performance: the residual bootstrap (thick dashed lines: $\sqrt{N}\left(\widehat{\phi}_{k}^{*}(v)-\right.$ $\left.\widehat{\phi}_{k}(v)\right)$ ) and the least squares estimators (thin lines: $\sqrt{N}\left(\widehat{\phi}_{k}(v)-\phi_{k}(v)\right)$ ) for M2 and sample size $n=300$.

of the distributions are not very well estimated, however this problem occurs also when bootstrapping i.i.d. data.

When a sample is small $n=30$ the distributions are not well estimated (see Figs. 7 and 8). Moreover, in this case we observe worse behaviour of the wild bootstrap method.

To determinate whether $\sqrt{N}\left(\widehat{\phi}_{k}^{*}(v)-\widehat{\phi}_{k}(v)\right)$ and $\sqrt{N}\left(\widehat{\phi}_{k}^{\dagger}(v)-\widehat{\phi}_{k}(v)\right)$ follow a normal distribution we use the Lilliefors test. The received $p$-values for M3 are gathered in Table 7. One can see that the $p$-values are larger than the significance level, the decision is to fail to reject the null hypothesis because we do not have enough evidence to conclude that the data do not follow a normal distribution. The Lilliefors test confirm that the data come from normal distribution when $n=300$, and we reject that hypothesis when $n=30$.

In our simulations we have considered $95 \%$ confidence intervals. Results gathered in Tables $1-3$ show that the actual coverage probabilities for simulated PAR models with $n=300$ are very well estimated. The results for both bootstrap methods are very similar but in most of the cases the ACP for all coefficients is closer to the nominal confidence level in the residual bootstrap method, especially when sample size is small (see 1). For M2, where we estimate relatively many parameters and additionally number of cycles is smaller comparing 


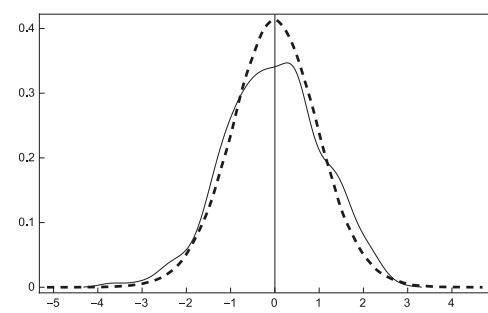

(a) $k=1, v=1$

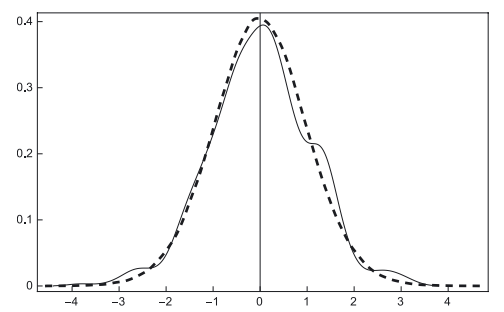

(d) $k=1, v=2$

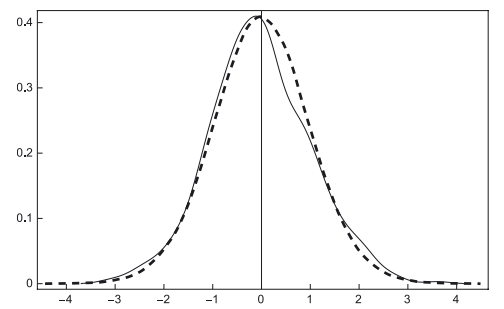

(g) $k=1, v=3$

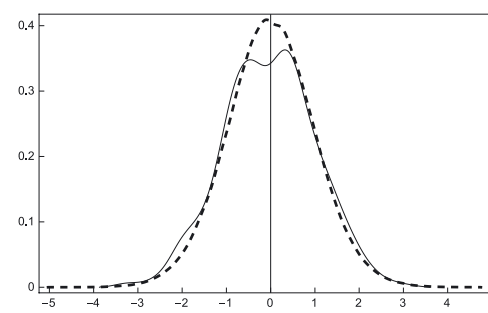

(b) $k=2, v=1$

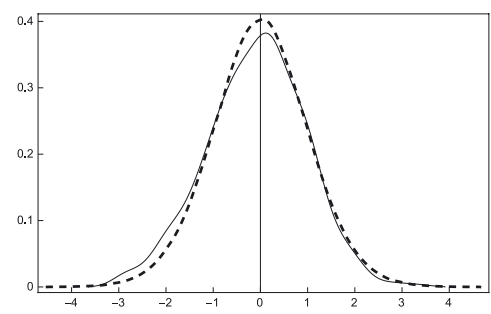

(e) $k=2, v=2$

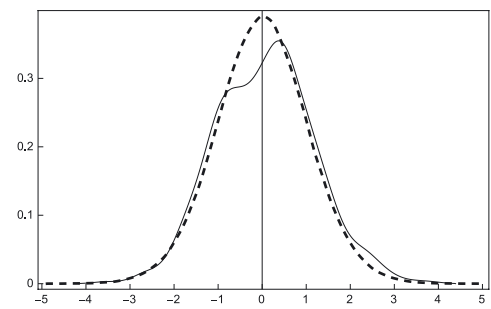

(h) $k=2, v=3$

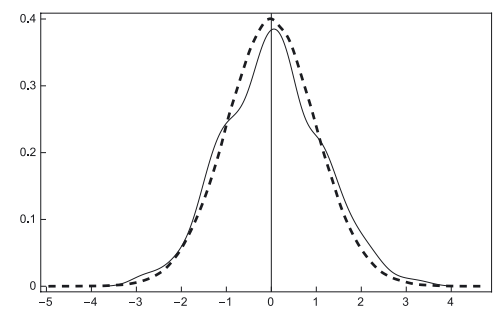

(c) $k=3, v=1$

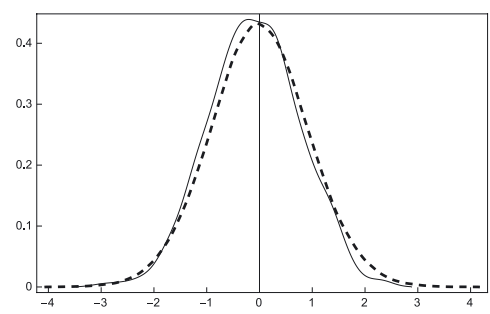

(f) $k=3, v=2$

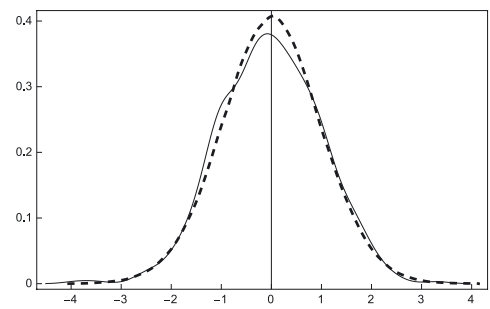

(i) $k=3, v=3$

Figure 4. Comparison of performance: the wild bootstrap (thick dashed lines: $\sqrt{N}\left(\widehat{\phi}_{k}^{\dagger}(v)-\right.$ $\left.\widehat{\phi}_{k}(v)\right)$ ) and the least squares estimators (thin lines: $\sqrt{N}\left(\widehat{\phi}_{k}(v)-\phi_{k}(v)\right)$ ) for M2 and sample size $n=300$.

with M1 or M3 of the same size $n=300$, the ACP is $3.8 \%$ lower than the nominal confidence level for the wild bootstrap method for $\phi_{2}(2)$ and $2.8 \%$ lower than the nominal confidence level for residual bootstrap method for coefficient $\phi_{1}(1)$. We obtain similar result for $\phi_{3}(3)$, this time ACPs are too large, but not larger by $2.8 \%$ than the nominal level. However, obtained results for M2 are still satisfactory.

\section{Conclusion}

In this paper, we have formulated two bootstrap procedures for periodically autoregressive time series. We have established a general limit results for bootstrap estimates of models' coefficients. We have shown that the residual and the wild bootstrap methods for periodic sequences is a natural generalization of bootstrap procedure for simple autoregressive processes given in [22]. Finally, we have illustrated our theoretical results by simulations. The simulations confirmed the weak consistency of the residual and the wild bootstrap for PAR sequences. 


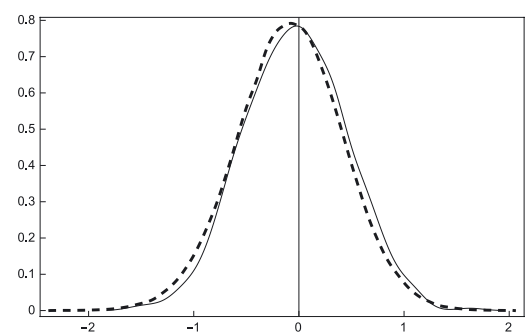

(a) $k=1, v=1$

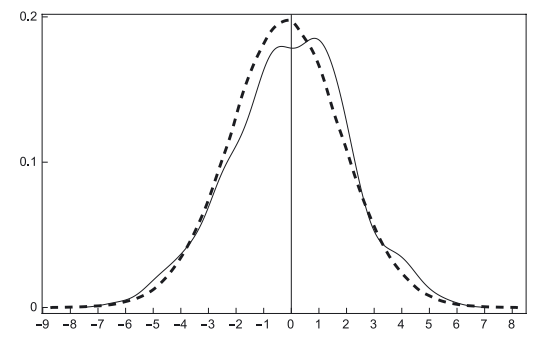

(c) $k=1, v=2$

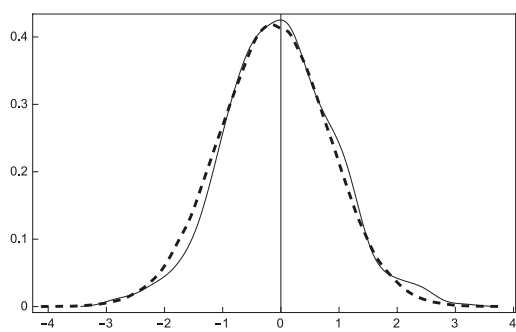

(b) $k=2, v=1$

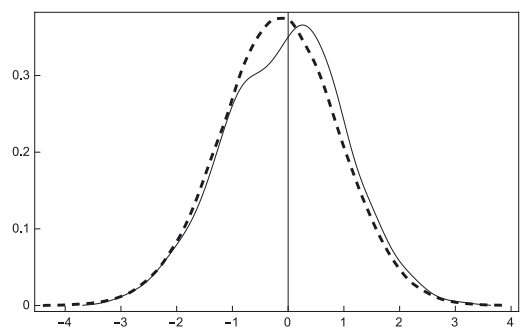

(d) $k=2, v=2$

FiguRE 5. Comparison of performance: the residual bootstrap (thick dashed lines: $\sqrt{N}\left(\widehat{\phi}_{k}^{*}(v)-\right.$ $\left.\left.\widehat{\phi}_{k}(v)\right)\right)$ and the least squares estimators (thin lines: $\left.\sqrt{N}\left(\widehat{\phi}_{k}(v)-\phi_{k}(v)\right)\right)$ for M3 and sample size $n=300$.

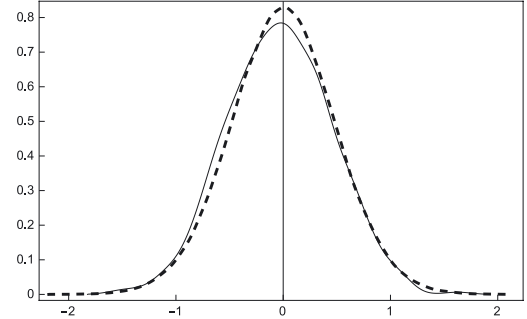

(a) $k=1, v=1$

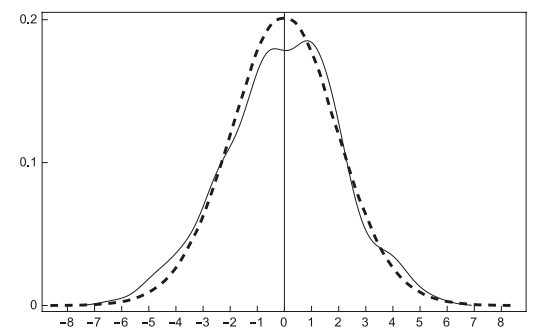

(c) $k=1, v=2$

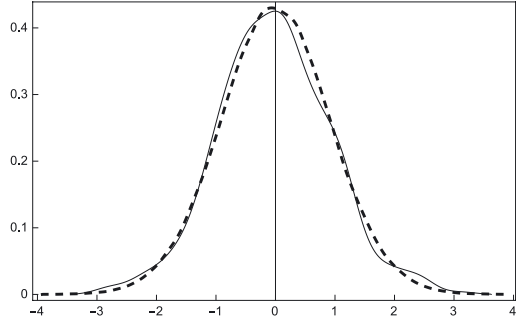

(b) $k=2, v=1$

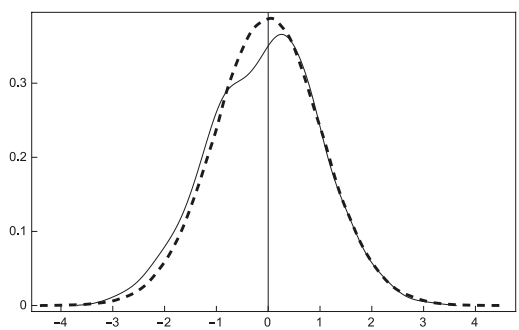

(d) $k=2, v=2$

FIGURE 6. Comparison of performance: the wild bootstrap (thick dashed lines: $\sqrt{N}\left(\widehat{\phi}_{k}^{\dagger}(v)-\right.$ $\left.\widehat{\phi}_{k}(v)\right)$ ) and the least squares estimators (thin lines: $\sqrt{N}\left(\widehat{\phi}_{k}(v)-\phi_{k}(v)\right)$ ) for M3 and sample size $n=300$. 


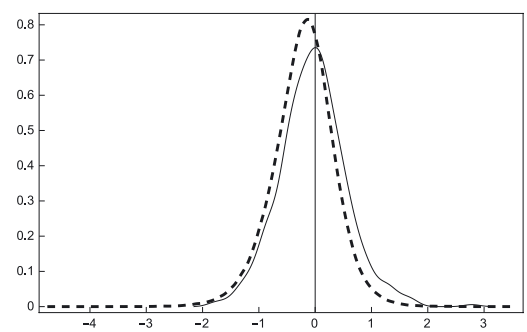

(a) $k=1, v=1$

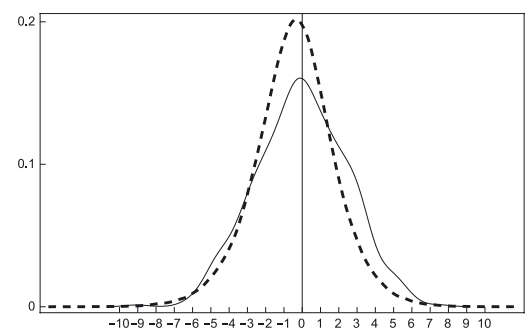

(c) $k=1, v=2$

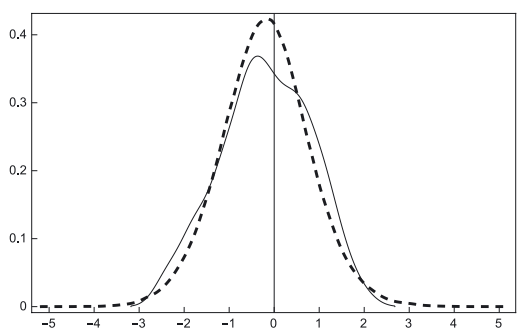

(b) $k=2, v=1$

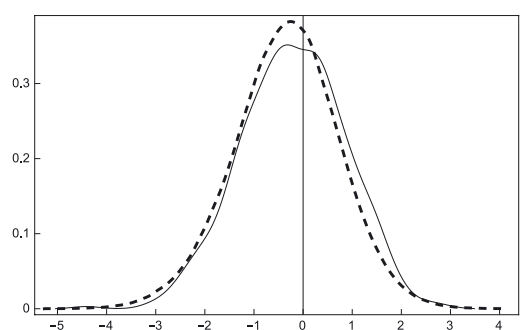

(d) $k=2, v=2$

FiguRE 7. Comparison of performance: the residual bootstrap (thick dashed lines: $\sqrt{N}\left(\widehat{\phi}_{k}^{*}(v)-\right.$ $\left.\left.\widehat{\phi}_{k}(v)\right)\right)$ and the least squares estimators (thin lines: $\left.\sqrt{N}\left(\widehat{\phi}_{k}(v)-\phi_{k}(v)\right)\right)$ for M3 and sample size $n=30$.

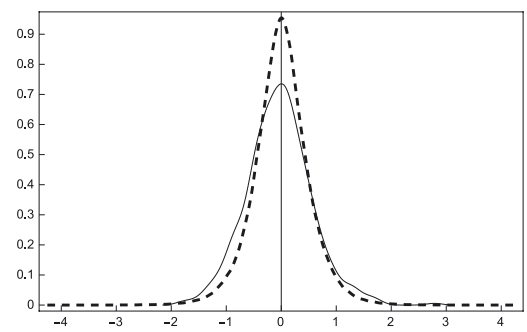

(a) $k=1, v=1$

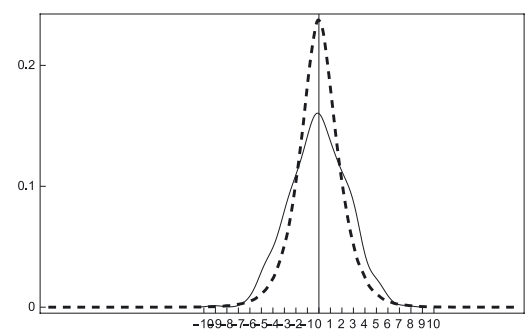

(c) $k=1, v=2$

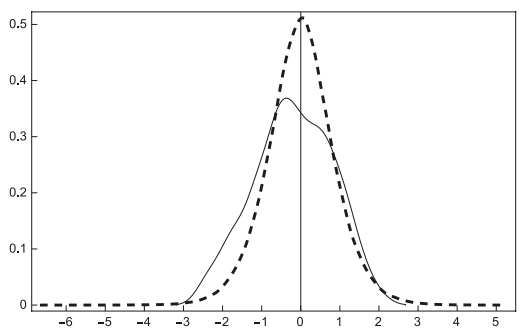

(b) $k=2, v=1$

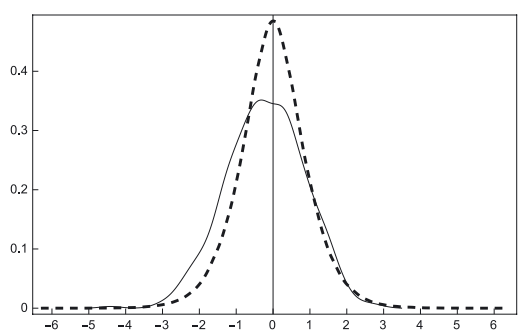

(d) $k=2, v=2$

FIGURE 8. Comparison of performance: the wild bootstrap (thick dashed lines: $\sqrt{N}\left(\widehat{\phi}_{k}^{\dagger}(v)-\right.$ $\left.\left.\widehat{\phi}_{k}(v)\right)\right)$ and the least squares estimators (thin lines: $\sqrt{N}\left(\widehat{\phi}_{k}(v)-\phi_{k}(v)\right)$ ) for M3 and sample size $n=30$. 
TABle 1. Actual coverage probabilities for M1. Rows 4 and 5 contain results for the residual and the wild bootstrap, respectively. For both methods ACPs when sample sizes $n=300$ are presented.

\begin{tabular}{|c|c|c|c|c|}
\cline { 3 - 5 } \multicolumn{2}{c|}{} & \multicolumn{3}{c|}{ ACP } \\
\cline { 3 - 5 } \multicolumn{2}{c|}{} & \multicolumn{3}{c|}{ M1 } \\
\hline bootstrap & $n$ & $\phi_{1}(1)$ & $\phi_{2}(1)$ & $\phi_{3}(1)$ \\
residual & 300 & $94.0 \%$ & $95.0 \%$ & $96.8 \%$ \\
wild & 300 & $93.8 \%$ & $94.6 \%$ & $95.8 \%$ \\
\hline
\end{tabular}

TABLE 2. Actual coverage probabilities for M2. Rows 4 and 5 contain results for the residual and the wild bootstrap, respectively. For both methods ACPs when sample sizes $n=300$ are presented.

\begin{tabular}{|c|c|c|c|c|c|c|c|c|c|c|}
\cline { 3 - 10 } \multicolumn{1}{c|}{} & \multicolumn{10}{c|}{ ACP } \\
\cline { 3 - 10 } \multicolumn{1}{c|}{} & \multicolumn{10}{c|}{ M2 } \\
bootstrap & $n$ & $\phi_{1}(1)$ & $\phi_{2}(1)$ & $\phi_{3}(1)$ & $\phi_{1}(2)$ & $\phi_{2}(2)$ & $\phi_{3}(2)$ & $\phi_{1}(3)$ & $\phi_{3}(3)$ & $\phi_{3}(3)$ \\
residual & 300 & $92.2 \%$ & $93.8 \%$ & $92.8 \%$ & $93.2 \%$ & $92.6 \%$ & $93.2 \%$ & $92.2 \%$ & $97.8 \%$ & $95.4 \%$ \\
wild & 300 & $91.6 \%$ & $94.0 \%$ & $92.6 \%$ & $93.6 \%$ & $91.2 \%$ & $92.2 \%$ & $92.4 \%$ & $97.0 \%$ & $94.2 \%$ \\
\hline
\end{tabular}

TABLE 3. Actual coverage probabilities for M3. Rows 4-5 and 6-7 contain results for the residual and the wild bootstrap, respectively. For both methods ACPs for two sample sizes $n=30$ and $n=300$ are presented.

\begin{tabular}{|c|c|c|c|c|c|}
\cline { 3 - 6 } \multicolumn{2}{c|}{} & \multicolumn{4}{c|}{ ACP } \\
\cline { 3 - 6 } \multicolumn{2}{c|}{} & \multicolumn{4}{c|}{ M3 } \\
\hline bootstrap & $n$ & $\phi_{1}(1)$ & $\phi_{2}(1)$ & $\phi_{1}(2)$ & $\phi_{2}(2)$ \\
\hline \multirow{2}{*}{ residual } & 30 & $85.8 \%$ & $86.0 \%$ & $87.4 \%$ & $92.8 \%$ \\
& 300 & $95.0 \%$ & $92.2 \%$ & $94.0 \%$ & $94.8 \%$ \\
\hline \multirow{2}{*}{ wild } & 30 & $85.4 \%$ & $83.6 \%$ & $85.4 \%$ & $84.0 \%$ \\
& 300 & $95.0 \%$ & $92.8 \%$ & $93.2 \%$ & $94.0 \%$ \\
\hline
\end{tabular}

TABLE 4. Average lengths of confidence intervals for M1. Rows 4 and 5 contain results for the residual and the wild bootstrap, respectively. For both methods the average lengths of confidence intervals when sample sizes $n=300$ are presented.

\begin{tabular}{|c|c|c|c|c|}
\cline { 3 - 5 } \multicolumn{2}{c|}{} & \multicolumn{3}{c|}{ average lengths of CI } \\
\cline { 3 - 5 } \multicolumn{2}{c|}{} & \multicolumn{3}{c|}{ M1 } \\
\hline bootstrap & $n$ & $\phi_{1}(1)$ & $\phi_{2}(1)$ & $\phi_{3}(1)$ \\
residual & 300 & 0.38057 & 0.38084 & 0.37138 \\
wild & 300 & 0.38456 & 0.38297 & 0.37376 \\
\hline
\end{tabular}

TABLe 5. Average lengths of confidence intervals for M2. Rows 4 and 5 contain results for the residual and the wild bootstrap, respectively. For both methods the average lengths of confidence intervals when sample sizes $n=300$ are presented.

\begin{tabular}{|c|c|c|c|c|c|c|c|c|c|c|}
\hline & \multicolumn{9}{|c|}{ average lengths of CI } \\
\hline & & \multicolumn{9}{|c|}{ M2 } \\
\hline bootstrap & $n$ & $\phi_{1}(1)$ & $\phi_{2}(1)$ & $\phi_{3}(1)$ & $\phi_{1}(2)$ & $\phi_{2}(2)$ & $\phi_{3}(2)$ & $\phi_{1}(3)$ & $\phi_{3}(3)$ & $\phi_{3}(3)$ \\
\hline residual & 300 & 0.38665 & 0.39142 & 0.38653 & 0.38419 & 0.39462 & 0.40491 & 0.39923 & 0.36842 & 0.39067 \\
\hline wild & 300 & 0.38390 & 0.39003 & 0.38654 & 0.38650 & 0.39608 & 0.40737 & 0.39400 & 0.36586 & 0.38926 \\
\hline
\end{tabular}


TABLE 6. Average lengths of confidence intervals for M3. Rows 4-5 and 6-7 contain results for the residual and the wild bootstrap, respectively. For both methods the average lengths of confidence intervals for two sample sizes $n=30$ and $n=300$ are presented.

\begin{tabular}{|c|c|c|c|c|c|}
\hline & \multicolumn{4}{|c|}{ Average lengths of CI } \\
\hline & & \multicolumn{4}{|c|}{ M3 } \\
\hline bootstrap & $n$ & $\phi_{1}(1)$ & $\phi_{2}(1)$ & $\phi_{1}(2)$ & $\phi_{2}(2)$ \\
\hline \multirow{2}{*}{ residual } & 30 & 0.47100 & 1.94341 & 0.86581 & 0.95234 \\
\hline & 300 & 0.15566 & 0.63470 & 0.29701 & 0.33056 \\
\hline \multirow{2}{*}{ wild } & 30 & 0.48486 & 1.97151 & 0.85201 & 0.91483 \\
\hline & 300 & 0.15580 & 0.63781 & 0.29656 & 0.32909 \\
\hline
\end{tabular}

TABLE 7. $p$-values of the Lilliefors test that determinate normality of $\sqrt{N}\left(\widehat{\phi}_{k}^{*}(v)-\widehat{\phi}_{k}(v)\right)$ and $\sqrt{N}\left(\widehat{\phi}_{k}^{\dagger}(v)-\widehat{\phi}_{k}(v)\right)$ for model M3.

\begin{tabular}{|c|c|c|c|c|c|}
\cline { 3 - 6 } \multicolumn{2}{c|}{} & \multicolumn{4}{c|}{$p$-values of normality test } \\
\cline { 3 - 6 } \multicolumn{2}{c|}{} & \multicolumn{4}{c|}{ M3 } \\
\hline bootstrap & $n$ & $\phi_{1}(1)$ & $\phi_{2}(1)$ & $\phi_{1}(2)$ & $\phi_{2}(2)$ \\
\hline \multirow{2}{*}{ residual } & 30 & 0.0138 & 0.5276 & 0.1332 & 0.0312 \\
& 300 & 0.4796 & 0.1746 & 0.9999 & 0.5738 \\
\hline \multirow{2}{*}{ wild } & 30 & 0.5314 & 0.8883 & 0.5915 & 0.7716 \\
& 300 & 0.8925 & 0.0899 & 0.5294 & 0.9267 \\
\hline
\end{tabular}

Acknowledgements. We thank Patrice Bertail for his remarks and useful comments regarding the content of this paper. We would also like to thank referees for their suggestions which greatly improved the presentation of this paper. The authors wish to acknowledge that this problem and initial guidance was due to Anna Dudek and Harry Hurd.

\section{REFERENCES}

[1] R. Arora, W. Sethares and J. Bucklew, Latent periodicities in genome sequences. IEEE J. Selected Topics Signal Proc. 2 (2008) 332-342.

[2] I.V. Basawa and R.B. Lund, Recursive prediction and likelihood evaluation for periodic ARMA models. J. Time Ser. Ana. 21 (2000) $75-93$.

[3] I.V. Basawa and R.B. Lund, Large sample properties of parameter for periodic ARMA models. J. Time Ser. Anal. 21 (2001) $75-93$.

[4] A. Bibi and I. Lescheb, A note on integrated periodic GARCH processes. Statist. Probab. Lett. 87 (2014) $121-124$.

[5] E. Broszkiewicz-Suwaj, A. Makagon, R. Weron and A. Wyłomańska, On detecting and modeling periodic correlation in financial data. Proceedings of the XVIII Max Born Symposium. Phys. A: Statist. Mech. Appl. 336 (2004) $196-205$.

[6] E. Carlstein, The use of subseries values for estimating the variance of a general statistics from a stationary sequence. Ann. Statist. 14 (1986) 1171-1179.

[7] V. Chan, S.N. Lahiri and W.Q. Meeker, Block bootstrap estimation of the distribution of cumulative outdoor degradation. Technometrics 46 (2004) 215-224.

[8] J. Dowell, S. Weiss, D. Hill and D. Infield, A cyclo-stationary complex multichannel Wiener filter for the prediction of wind speed and direction. Proc. 21st Europ. Signal Proc. Confer. (EUSIPCO 2013).

[9] A.E. Dudek, J. Leśkow, E. Paparoditis and D. Politis, A generalized block bootstrap for seasonal time series. J. Time Ser. Anal. 35 (2014) 89-114.

[10] W. Gardner and L.E. Franks, Characterization of cyclostationary random signal processes. IEEE Trans. Inform. Theory 21 (1975) 4-14.

[11] C. Gaucherel, Analysis of ENSO interannual oscillations using non-stationary quasi-periodic statistics. A study of ENSO memory. Int. J. Climatology 30 (2010) 926-934.

[12] F. Ghaderi, K. Nazarpour, J.G. McWhirter and S. Sanei, Removal of ballistocardiogram artifacts using the cyclostationary source extraction method. IEEE Trans. Biomed. Eng. 57 (2010) 2667-2676.

[13] E.G. Gladyšhev, Periodically correlated random sequences. Soviet Math. 2 (1961) 385-388.

[14] P. Hall, Resampling a coverage pattern. Stoch. Process. Appl. 20 (1985) 231-246.

[15] P. Hall, The Bootstrap and Edgeworth Expansion. New York: Springer Verlag (1992).

[16] J.D. Hamilton, Time Series Anal. New Jersey, Princeton University Press (1994). 
[17] B. Iqelan, Periodically Correlated Time Series: Models and Examples. Lambert Academic Publishing (2011).

[18] R.H. Jones and W.M. Brelsford, Time series with periodic structure. Biometrika 54 (1967) 403-408.

[19] K.-Y. Kim, B. Hamlington and H. Na, Theoretical foundation of cyclostationary EOF analysis for geophysical and climatic variables. Concepts and examples. Earth-Sci. Rev. 150 (2015) 201-218.

[20] J. Kreiss and S. Lahiri, Bootstrap Methods for Time Series. Time Ser. Anal.: Methods Appl. 30 (2012) 3-23.

[21] H. Künsch, The jackknife and the bootstrap for general stationary observations. Ann. Statist. 17 (1989) 1217-1241.

[22] S. Lahiri, Resampling methods for Dependent Data. Springer Verlag (2003).

[23] R. Liu, Bootstrap Procedures under some Non-I.I.D. Models. Ann. Statist. 164 (1988) 1696-1708.

[24] R.Y. Liu and K. Singh, Moving blocks jackknife and bootstrap capture weak dependence. Exploring the Limits of Bootstrap, edited by R. LePage and L. Billard. Wiley New York (1992) 225-248.

[25] S. Maiz, El. Badaoui, F. Bonnardot and C. Serviere, New second order cyclostationary analysis and application to the detection and characterization of a runner's fatigue. Signal Processing 102 (2014) 188-200.

[26] A.S. Monin, Stationary and periodic time series in the general circulation of the atmosphere, in: Proc. Symp. Time Ser. Anal., edited by M. Rosenblatt. John Wiley and Sons, New York (1963) 144-151.

[27] M. Pagano, On periodic and multiple autoregressions. Ann. Statist. 6 (1978) 1310-1317.

[28] E. Parzen and M. Pagano, An approach to modeling seasonally stationary time series. J. Econom. 9 (1979) $137-153$.

[29] D.N. Politis, Resampling time series with seasonal components, in Frontiers in Data Mining and Bioinformatics: Proceedings of the 33rd Symposium on the Interface of Computing Science and Statistics, Orange County, California 13-17 (2001) 619-621.

[30] Q. Shao and P.P. Ni, Least-squares estimation and ANOVA for periodic autoregressive time series. Statist. Probab. Lett. 69 (2004) 287-297.

[31] K. Shimizu, Boostrapping stationary ARMA-GARCH Models. Vieweg+Teubner Research (2009).

[32] G.B. Thomas and D.F. Fiering, Mathematical synthesis of streamflow sequences for the analysis of river basins by simulation, in: Design of Water Resources Syst., edited by A. Maas. Harvard University Press, Cambridge (1962).

[33] E. Ursu and P. Duchesne, On modelling and diagnostic checking of vector periodic autoregressive time series models. J. Time Seri. Anal. 30 (2009) 70-96.

[34] A. Vecchia, Maximum Likelihood Estimation for Periodic Autoregressive Moving Average Models. Technometrics 27 (1985) 375-384. 